\title{
REFORZANDO LAS CAPACIDADES EN INVESTIGACIÓN EN INFORMÁTICA PARA LA SALUD GLOBAL EN LA REGIÓN ANDINA A TRAVÉS DE LA COLABORACIÓN INTERNACIONAL
}

\author{
Walter H. Curioso ${ }^{1,2, a, b}$, Patricia J. García1,3,a , Greta M. Castillo ${ }^{4, c}$, Magaly M. Blas ${ }^{1, a, d}$, \\ Amaya Perez-Brumer ${ }^{1, \mathrm{e}}$, Mirko Zimic ${ }^{5, \mathrm{f}}$, Red QUIPU ${ }^{\dagger}$
}

\begin{abstract}
RESUMEN
Para mejorar la salud global y bienestar de una población se requiere de recursos humanos capacitados, no solo en el campo de la medicina y salud, sino también en el campo de la informática. Desafortunadamente, los programas de entrenamiento e investigación en informática biomédica en países en desarrollo son escasos y poco documentados. El objetivo del presente trabajo es reportar los resultados del primer Taller Internacional de Expertos en Informática para la región andina que se llevó a cabo en marzo de 2010 en Lima y que incluye la descripción de nueve casos de estudio procedentes de instituciones de América Latina. En el taller participaron 23 expertos latinoamericanos, quienes discutieron la necesidad de entrenamiento e investigación multidisciplinaria en informática biomédica en áreas prioritarias para América Latina. Además, se estableció la Red QUIPU debido a la necesidad de ampliar y consolidar una red de investigación y entrenamiento a nivel regional y global.
\end{abstract}

Palabras clave: Capacitación; Investigación biomédica; Informática médica; Cooperación horizontal; América Latina (fuente: DeCS BIREME).

\section{STRENGTHENING GLOBAL HEALTH INFORMATICS RESEARCH WITHIN THE ANDEAN REGION THROUGH INTERNATIONAL COLLABORATION}

\begin{abstract}
To improve global health and the welfare of a population, skilled human resources are required, not only in medicine and health, but also in the field of informatics. Unfortunately, training and research programs specific to biomedical informatics in developing countries are both scarce and poorly documented. The aim of this paper is to report the results from the first Informatics Expert Meeting for the Andean Region, including, nine Latin American based institutional case studies. This two-day event occurred in March 2010 and brought together twenty-three leaders in biomedical informatics from around the world. The blend of practical and experiential advice from these experts contributed to rich discussions addressing both challenges and applications of informatics within Latin American. In addition, to address the needs emphasized at the meeting, the QUIPU Network was established to expand the research consortium in the Andean Region, Latin America, and internationally. The use of these new technologies in existing public health training and research programs will be key to improving the health of populations in the Andean Region and around the globe.
\end{abstract}

Key words: Training; Biomedical research; Medical informatics; Horizontal cooperation; Latin America (source: MeSH NLM).

\section{INTRODUCCIÓN}

Para mejorar la salud global y bienestar de una población se requiere, entre otros factores, de recursos humanos capacitados, no solo en el campo de la medicina y salud, sino también en el campo de la informática, ya que hoy en día, la tecnología puede permitir mejoras sustanciales en diversos aspectos ${ }^{(1)}$. Las tecnologías de la información y comunicación (TIC) tienen el potencial de beneficiar a países desarrollados y países en desarrollo. La Organización Mundial de la Salud identificó al uso de las TIC como habilidad prioritaria para el desarrollo de los recursos humanos en salud ${ }^{(2)}$. Además, el uso de las TIC se reconoce cada vez más como un elemento crucial para la mejora de los sistemas de salud y como un medio para poder llegar a alcanzar los Objetivos de Desarrollo del Milenio ${ }^{(2)}$.

\footnotetext{
Facultad de Salud Pública y Administración, Universidad Peruana Cayetano Heredia. Lima, Perú.

Biomedical and Health Informatics, School of Medicine, University of Washington. Seattle, Washington, USA.

Department of Global Health, University of Washington, Seattle, WA, USA.

Health Information Science. University of Victoria. British Columbia, Canada.

5 Unidad de Bioinformática-Laboratorios de Investigación y Desarrollo. Facultad de Ciencias y Filosofía, Universidad Peruana Cayetano Heredia. Lima, Perú.

a Médico, Maestro en Salud Pública; ${ }^{\text {b }}$ Especialista en Informática Biomédica y Candidato al Doctorado en Informática Biomédica; ${ }^{c}$ Maestranda en

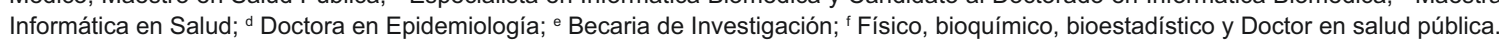

†er sección "Miembros de la Red QUIPU" para la lista completa de participantes de la Red.
}

Recibido: 17-08-10 Aprobado: 08-09-10 
Así, el desarrollo e implementación de programas de entrenamiento e investigación en informática biomédica se convierte en necesidades cruciales para crear una masa crítica de profesionales adecuadamente capacitados que respondan a las necesidades locales y regionales. Entendemos la informática biomédica como una disciplina científica interdisciplinaria que trata sobre la información biomédica, los datos y el conocimiento -su almacenamiento, recuperación, modelamiento y uso óptimo para la resolución de problemas, toma de decisiones, prevención y promoción de la salud (3). En este contexto, podemos subdividir a la informática biomédica en dos grandes áreas: informática en salud (ejemplo: aplicaciones clínicas y en salud pública) y la bioinformática (ejemplo: aplicaciones en procesos moleculares y celulares).

Además, la colaboración internacional como un fenómeno propio a la globalización en salud, se constituye en un componente fundamental para el éxito y sostenibilidad de muchos programas de entrenamiento e investigación en informática biomédica ${ }^{(4,5)}$. Un ejemplo de colaboración entre países desarrollados es el International Partnership for Health Informatics Education (IPHIE) que es una asociación que busca promover la educación a través de la colaboración entre Europa y los Estados Unidos con programas de capacitación para estudiantes de posgrado y pregrado en medicina e informática en salud (http://www.iphie.org).

Desafortunadamente, los programas de entrenamiento e investigación en informática biomédica en países en desarrollo son escasos y poco documentados. Sin embargo, se ha desarrollado algunas iniciativas sostenibles. Por ejemplo, Brasil ha venido trabajando programas en informática en salud en las últimas décadas, donde se han desarrollado cursos e investigaciones colaborativas entre instituciones como la Universidad de Sao Paulo y la Universidad de Harvard (6). Esta colaboración interinstitucional ha sido promovida por el programa Informatics Training for Global Health (7) del John E. Fogarty International Center (FIC) de los Institutos Nacionales de Salud de los Estados Unidos, centro establecido en 1968 para la promoción de la salud global (http://www.fic.nih.gov).

En Perú, la Universidad Peruana Cayetano Heredia (UPCH) en el año 2009, recibió financiamiento del FIC para desarrollar el proyecto QUIPU para la promoción de investigación y la formación de profesionales en informática biomédica y salud global, y que busca convertirse en un Centro de Excelencia de la más alta calidad en la región andina. Quipu, que significa nudo en quechua, es un sistema ancestral usado por los incas en los Andes para registrar y distribuir información. El proyecto QUIPU (http://www.andeanquipu.org) es liderado por la UPCH con la participación del U.S. Naval Medical Research Center Detachment (NMRCD) en Lima, la Universidad del Cauca en Colombia y la Universidad de Washington en Seattle (USA).

Los objetivos del Proyecto QUIPU son:

- Desarrollar e implementar oportunidades de entrenamiento a corto y largo plazo, en informática biomédica para la salud global en la región andina;

- Comprometer a nuevos investigadores en la región andina, para realizar investigaciones en informática en salud y bioinformática; y

- Expandir y consolidar una red de investigación en la región andina, promoviendo la colaboración sur-sur, y de las universidades colaboradoras en USA y otras instituciones.

Recientemente, el proyecto QUIPU desarrolló el primer taller de expertos latinoamericanos en informática biomédica. El objetivo del presente trabajo es reportar los resultados del primer taller internacional de expertos en informática para la región andina que se llevó a cabo en Lima y que incluye la descripción de experiencias sobre entrenamiento e investigación en informática biomédica con casos de estudio.

\section{TALLER DE EXPERTOS}

El "Taller de Expertos Latinoamericanos en Informática para la Salud Global" se llevó a cabo el 26 y 27 de marzo de 2010 en Lima, Perú. Expertos de América Latina y los Estados Unidos se reunieron para compartir experiencias, con la intención de desarrollar relaciones de colaboración, generar ideas sobre las necesidades y prioridades de investigación y discutir las necesidades de entrenamiento en informática biomédica.

En el taller participaron 23 expertos de América Latina (Perú, Colombia, Argentina, Uruguay, Venezuela, Ecuador y Chile) y de Estados Unidos, en el cual se reunieron para discutir las prioridades de currículo e investigación y las posibilidades de colaboración en América Latina. La discusión se enfocó en las siguientes áreas: problemas y necesidades con respecto a la investigación y entrenamiento en informática biomédica en la región andina y América Latina; currículo en informática biomédica para la región, y el trabajo multidisciplinario en informática biomédica. La conferencia concluyó con un resumen sobre lo que fue discutido en los días previos. Detalles el evento se encuentra disponible en el siguiente enlace: http://www. andeanquipu.org/index.php?option=com_content\&view $=$ article $\& i d=51 \&$ ltemid $=74 \&$ lang $=e s$. 


\section{CASOS DE ESTUDIO}

A continuación se presenta un resumen de los nueve casos presentados por los participantes latinoamericanos.

\section{INFORMÁTICA EN SALUD EN EL PERÚ: EL PROGRAMA AMAUTA}

En Perú, el campo de la informática biomédica está emergiendo ${ }^{(8)}$. Uno de los programas de entrenamiento en informática biomédica que merece destacar es el programa AMAUTA, desarrollado para capacitar a profesionales de la salud en la aplicación de la informática para la salud (9). En este programa colaborativo participaron la Universidad Peruana Cayetano Heredia (UPCH), la Universidad Nacional Mayor de San Marcos y la Universidad de Washington (UW) en Seattle, Washington, USA, con el apoyo del FIC ${ }^{(9)}$. El programa fomentó el desarrollo de la Unidad de Informática en Salud en la UPCH constituida por un equipo multidisciplinario de profesionales dedicados a la investigación y desarrollo de las TIC aplicados a la salud.

Durante el período 1999-2009, se organizó en Lima cuatro cursos cortos en los años 2000, 2001, 2005 y 2008 en los cuales se entrenó a más de 200 profesionales de la salud (10-12). Los dos primeros cursos trataron temas referentes a la informática médica y la informática de salud pública. Los dos últimos cursos trataron temas referentes a la informática médica, informática de salud pública y bioinformática. Desde el año 2005, se agregaron temas sobre bioinformática debido a la creciente demanda de entrenamiento en bioinformática en el Perú (11).

Además, se entrenó a profesionales peruanos a nivel de maestrías y doctorados en la UW. En el año 2009, la red de colaboradores se expandió y se convirtió en el proyecto QUIPU. La UPCH promueve la colaboración efectiva sur-sur en el adiestramiento y la investigación en informática biomédica. Una estrategia, actualmente en desarrollo, es un Centro de Excelencia para el entrenamiento e investigación en informática biomédica para la región andina y de América Latina.

\section{BIOINFORMÁTICA EN EL PERÚ: EL CASO DE LA UPCH}

El programa de entrenamiento e investigación en bioinformática en la UPCH se sustenta en las actividades académicas y de investigación de la Unidad de Bioinformática de la Facultad de Ciencias y Filosofía. Estas actividades han venido realizándose desde el año 2003 hasta la fecha. El desarrollo de la bioinformática en la UPCH corresponde fundamentalmente al uso y al desarrollo de herramientas especializadas aplicadas a resolver problemas diversos tanto en ciencia básica como aplicaciones en salud. El equipo de investigación de la Unidad de Bioinformática está integrado por físicos, químicos, matemáticos, bioestadísticos, ingenieros y biólogos. Desafortunadamente, esta interacción se ve limitada por una barrera de "lenguaje" entre los profesionales de las ciencias biomédicas y los científicos/ingenieros no-biológicos, como consecuencia de las actuales currículas. Esta barrera ha venido disminuyendo en los últimos años con la participación más activa y colaborativa entre estudiantes selectos de distintas instituciones. Así, cada vez con mayor frecuencia se encuentra que estudiantes de diferentes disciplinas están comenzando a trabajar juntos.

Los cursos sobre la bioinformática ofrecidos en la UPCH incluyen los temas de análisis de secuencia, modelamiento molecular, química computacional, ingeniería de proteínas y enzimología; más recientemente ensamblaje y anotación de genomas. Además, se tiene diversos cursos complementarios que incluyen las matemáticas, la física, la química, la informática y la programación como curso avanzado. Los futuros cursos organizados por el proyecto QUIPU incluirán: diseño avanzado racional de los medicamentos, bioinformática avanzada y biotecnología en la biomedicina. Los cursos integrados son fundamentales en la bioinformática, biotecnología, informática y salud. El entrenamiento y la investigación en bioinformática involucran a un equipo multidisciplinario en el que cada individuo tiene diferentes habilidades y capacidades, con el fin de permitirles trabajar en proyectos de salud, uno de ellos es la aplicación del reconocimiento matemático de patrones y análisis de imágenes para el soporte del telediagnóstico de la tuberculosis y el desarrollo de una plataforma con tecnologías móviles para la transmisión de información (13).

\section{INFORMÁTICA MÉDICA EN COLOMBIA: EL CASO DE LA UNIVERSIDAD DE ANTIOQUIA Y LA UNIVERSIDAD DEL CAUCA}

La Universidad de Antioquia ofrece una Maestría en Ciencias Biomédicas con énfasis en informática médica, la cual es actualmente ofrecida exclusivamente a profesionales de la salud. Sin embargo, la universidad está planeando ofrecer un programa de maestría en informática de la salud a partir del 2011, el cual podrá ser cursado por profesionales de otras disciplinas. Los cursos ofrecidos en este programa incluyen: introducción a la informática para la salud, telemedicina, representación del conocimiento biomédico, y diseño de interfaces gráficas para e-Salud. La Universidad de Antioquia ofrece también un certificado de posgrado en informática médica el cual es apoyado por el Centro de Ciencias de la Salud de la Universidad de Texas en Houston. 
La Universidad del Cauca, por su parte, no cuenta con un programa oficial de posgrado en informática biomédica, pero ofrece una Maestría y un Doctorado en Ingeniería Telemática, los cuales cuentan con un área de investigación en e-Salud. Este programa es ofrecido exclusivamente a profesionales de la ingeniería, y es apoyado por el eHealth Competence Center Regensburg en Alemania (http://www.ehcc.de). Como resultado del proyecto QUIPU, la Universidad del Cauca planea ofrecer, a corto plazo, una maestría en Ciencias Biomédicas, la cual incluirá una línea de énfasis en informática biomédica y, a mediano plazo, una maestría en Informática de Salud. Las actividades de investigación en informática biomédica en Colombia están respaldadas por más de 50 grupos de investigación en áreas relacionadas con la informática para la salud, bioinformática y bioingeniería. Cabe destacar los desarrollos en telemedicina logrados por la Universidad Nacional en Bogotá, la Universidad de Caldas, la Fundación Cardiovascular de Colombia y la Universidad del Cauca ${ }^{(14)}$

\section{INFORMÁTICA EN SALUD DE LA UNIVERSIDAD JAVERIANA EN COLOMBIA}

El proyecto ENRICH: Enhancing Research and Informatics Capacity for Health Information in Colombia, establece una colaboración internacional entre la Universidad de Pittsburgh y la Universidad Javeriana en Bogotá, con el objetivo de incrementar la capacitación en el campo de la informática en salud, enfocándose en la investigación clínica. Así, se ha establecido una asociación con el Departamento de Epidemiología Clínica y Bioestadística (DCEB) de la Universidad Javeriana. Dentro de este departamento, se desarrollará un centro de informática médica como recurso regional para el entrenamiento en el país y como centro de apoyo para el programa ENRICH. La Universidad de Pittsburgh, con su experiencia en los temas de informática biomédica, construirá un programa integrado para educar a un grupo de investigadores en informática de salud y en métodos avanzados de investigación pertinentes a las necesidades de salud en Colombia.

El objetivo de este programa es establecer la infraestructura para la creación de un programa de entrenamiento formal en informática médica, bajo los auspicios de la DCEB en la Universidad Javeriana. Además, se proponen desarrollar aplicaciones informáticas en una serie de proyectos en el área de la investigación en trauma, como parte de proyectos que viene financiados por el FIC en Colombia desde el 2006. Estos estudios podrían tener un potencial impacto frente a los problemas de salud pública en Colombia.
INFORMÁTICA MÉDICA EN ARGENTINA: EL CASO DEL HOSPITAL ITALIANO DE BUENOS AIRES (HIBA)

En 1998, el HIBA comenzó a implementar un sistema de información de salud, con el cual actualmente se maneja toda la información relacionada con la asistencia sanitaria clínica y administrativa. Como parte del proyecto denominado Itálica, se creó un Departamento de Informática en Salud en el 2001 con la participación de más de 110 personas, entre ellas médicos, profesionales de tecnologías de información y técnicos. En el año 2000 se crea el Programa de Residencia de Informática Médica, de cuatro años de formación, con el objetivo de desarrollar recursos humanos en la informática médica (15). El objetivo principal del programa es capacitar a los médicos para ser el enlace entre la asistencia sanitaria y las tecnologías de la información en el ciclo de vida de sistemas de información sanitaria. Los graduados de la residencia son líderes en su campo.

El curso "Sistemas de información en los sistemas de salud: Introducción a la Informática Biomédica" se enmarca dentro del proyecto $10 \times 10$ coordinado por la Asociación Americana de Informática Médica (AMIA) ${ }^{(16)}$. La finalidad del curso es introducir a las personas que trabajan en distintas organizaciones de la salud en el complejo mundo de los sistemas de información, y que conozcan cómo las tecnologías de la información colaboran en mejorar la calidad, seguridad y costo-efectividad de la atención médica. El curso se dicta sobre una plataforma de e-learning (16). Las modalidades de enseñanza empleadas en el curso, incluyen conferencias, paneles de discusión, lecturas recomendadas y autoevaluaciones. Hasta agosto de 2010 más de 600 alumnos se han enrolado en el curso.

\section{INFORMÁTICA EN SALUD EN URUGUAY}

El proyecto FEMI, que es apoyado por el Banco Interamericano de Desarrollo, tiene como objetivo mejorar la eficiencia en la atención de la salud mediante el uso de las TIC ${ }^{(17)}$. Para lograr esto, es necesario que haya coordinación interdisciplinaria en cada una de las 23 instituciones de FEMI y el centro de referencia central. Hasta el año 2010 se formaron masivamente diferentes perfiles profesionales. Además, se implementó una estrategia basada en un entrenamiento enfocado al usuario final, tomando en cuenta la necesidad de profesionales capacitados (eventualmente 2800 médicos y más de diez mil empleados). El curso introductorio $10 \times 10$ en línea basado en los sistemas de información de salud (aproximadamente 150 horas de curso), fue desarrolladlo por AMIA y adaptado para el Hospital Italiano de Buenos Aires. En total, 72 profesionales tomaron el curso entre 2008 y 2009. En 
conclusión, el entrenamiento en informática fue posible en Uruguay a través de la colaboración con instituciones académicas de nivel nacional y regional, utilizando modelos presenciales y modalidades de aprendizaje en línea. Es necesario formar líderes capacitados para implementar exitosamente y manera amplia los sistemas de información clínica ${ }^{(17) .}$

\section{INFORMÁTICA EN SALUD EN VENEZUELA}

En Venezuela, específicamente en el Estado Zulia, el primer programa de informática biomédica entrenó a 300 personas, incluyendo profesionales de salud de varias áreas (médicos, nutricionistas, enfermeras, técnicos radiólogos, administradores de salud, etc.), en el marco de un proyecto de Automatización de Instituciones de Salud del Estado. En marzo de 2010, se realizó el primer diplomado que se encuentra administrado por el instituto IUTEPAL, junto a la Asociación Venezolana de Informática en Salud. El diplomado presentó a los estudiantes con conocimientos prácticos y experiencia para abordar los desafíos y proveer soluciones eficaces. A través del conjunto de habilidades técnicas y científicas, los estudiantes se convertirán en profesionales de éxito y líderes de procesos de cambio en el campo de la informática biomédica.

El diplomado busca involucrar a los estudiantes en la informática biomédica y ofrece visitas guiadas a diferentes centros. Los temas tratados en el diplomado incluyen: diseño de aplicaciones en salud, gestores de implementación de aplicaciones, desarrollo de sistemas de código abierto, mantenimiento técnico de los equipos (hardware y software), ingeniería de redes y cómo asumir el liderazgo en los centros de salud para organizar el cambio en el ámbito tecnológico. Muchos de los graduados tienen ahora una función importante dentro de las instituciones que están involucradas en numerosos proyectos de automatización en el ámbito hospitalario. Otros graduados se han convertido en líderes en los sectores de la salud e ingeniería. Los graduados capacitados en informática biomédica incluyen a médicos que se encuentran trabajando como directores de hospitales, jefes de los servicios de áreas clínicas, residentes, enfermeras, técnicos y personal de apoyo.

\section{INFORMÁTICA EN SALUD EN ECUADOR}

La informática en salud en Ecuador está emergiendo, promovida por universidades y por la Asociación Ecuatoriana de Informática Médica (inactiva desde hace cuatro años). Se ha desarrollado proyectos en telesalud por más de diez años, en varias provincias para llevar salud a comunidades remotas y crear enlaces educativos y asistenciales. La Universidad de
San Francisco de Quito, ofrece Informática de la Salud para los estudiantes de medicina, como parte la carrera dentro del curso de investigación. Otros programas en la universidad ofrecen cursos de telemedicina. Existen proyectos de colaboración en telemedicina y telesalud en los Andes del Ecuador.

Otros proyectos de colaboración que usan software basado en web para teleconsultas y para el entrenamiento a profesionales en medicina y promotores de salud de la comunidad, se encuentran en curso en las provincias de Chimborazo, Cotopaxi y próximamente en Esmeraldas. El curso en telemedicina incluye interconectividad y redes.

Existen planes para involucrar la telemedicina en las actividades curriculares de pregrado y posgrado, coordinadas con el Ministerio de Salud y aplicadas a las prácticas rurales y rotaciones de los médicos en hospitales públicos. Los planes futuros incluirán una maestría de telemedicina e informática médica que involucrará la colaboración virtual con instituciones internacionales.

\section{INFORMÁTICA BIOMÉDICA EN CHILE}

La informática biomédica es una disciplina que en Chile se ha desarrollado de manera poco organizada. La mayoría de las iniciativas en este campo han sido motivadas por la necesidad de implementar este tipo de tecnologías en lugar de ser el producto de una planificación cuidadosa. De la misma manera, la formación de recursos humanos con conocimientos avanzados en la disciplina ha sido escasa, con poca inversión en la producción de especialistas. Hasta la fecha de esta publicación, solo existe un programa técnico, de entrenamiento a nivel de pregrado en informática biomédica en el país. Dicho programa, de cuatro años de duración, es dictado por el Instituto Profesional y Centro de Formación Técnica DuocUC y el título ofrecido es el de Tecnólogo en Informática Biomédica. La segunda iniciativa, actualmente en fase de planificación, ha sido liderada por académicos de la Universidad de Heidelberg, la Universidad de Chile y la Pontificia Universidad Católica de Chile. A partir del año 2011 el Centro Heidelberg para América Latina, ubicado en Santiago de Chile, ofrecerá cursos de verano y una Maestría en Informática Biomédica. Dicho programa tendrá dos especializaciones, la primera estará centrada en la Informática en Salud -con énfasis en la gestión de la información sanitaria- y la segunda estará centrada en la bioinformática y el procesamiento de imágenes biomédicas -con énfasis en el procesamiento de la información y técnicas computacionales y estadísticas complejas-. Los alumnos podrán contar con doble titulación en Chile y en Alemania lo que permitirá también la internacionalización de sus egresados ${ }^{(18)}$. 


\section{TALLERES DE DISCUSIÓN SOBRE ENTRENAMIENTO Y PRIORIDADES DE INVESTIGACIÓN EN INFORMÁTICA BIOMÉDICA EN LA REGIÓN ANDINA Y AMÉRICA LATINA}

El debate en grupo se estructuró en las siguientes áreas: necesidades y problemas en materia de formación en informática biomédica en la región andina y América Latina; cuestiones específicas acerca de los planes de estudios y el nivel de interacción multidisciplinaria. Los participantes fueron divididos en dos grupos en los que tenían que responder a preguntas preestablecidas.

\section{NECESIDADES Y PROBLEMAS EN EL ENTRENAMIENTO EN INFORMÁTICA BIOMÉDICA}

Uno de los principales problemas identificados fue que no hay suficientes fondos para la educación en informática biomédica y estos programas son costosos. Si bien la demanda de estudiantes está aumentando, existe una falta de programas bien establecidos y materiales de calidad en nuestro idioma. No existe suficiente difusión y comunicación a nivel global respecto a los programas educativos que existen en la región andina y América Latina, entre facultades e instituciones a nivel local o regional. Existe una falta de acuerdo con las nomenclaturas. Se requiere de una estandarización en los elementos básicos de la educación y de definiciones. La homologación de créditos entre programas de diferentes países latinoamericanos es un desafío por enfrentar. Debería tenerse en cuenta iniciativas como la del Espacio Latinoamericano y del Caribe de Educación Superior (http://www.oui-iohe.org/ campus/eles/eles/). Por último, no existen iniciativas gubernamentales con visión a largo plazo (agravada por la alta rotación de funcionarios del gobierno). Para superar estos obstáculos es necesario tener en estos programas, recursos humanos bien calificados para la enseñanza. Otra estrategia es adaptar a nuestro medio los recursos y programas ya existentes, como la Acreditación Europea de Manejo de Ordenador (European Computer Driving Licence, ECDL).

Además, la mayoría de programas se basan solo en la teoría, de tal manera que existe una necesidad de incorporar prácticas $y$, cuanto más sea posible, que el estudiante tenga la oportunidad de desarrollar trabajo de campo y de socializar los resultados de sus investigaciones. Dado que muchos de los estudiantes proceden de diferentes disciplinas, es necesario definir de manera específica el perfil del estudiante. También es importante crear alianzas para trabajar juntos y lograr una mejor comunicación.

\section{CONOCIMIENTOS, HABILIDADES Y COMPETENCIAS PARA EL ENTRENAMIENTO}

Con el fin de mejorar la investigación y la formación de profesionales en informática biomédica en la región andina y América Latina, los conocimientos, habilidades y competencias que se necesitan dependen de la realidad de nuestro entorno. Por lo tanto, se debe definir cuidadosamente los requisitos en base a los problemas locales. Se debe desarrollar aplicaciones prácticas, similares al estilo europeo de aprendizaje, que hace hincapié en la investigación. Por ejemplo, se puede crear un programa de residentes en informática clínica o una residencia con formación de estudiantes no médicos. Además, debemos ser capaces de tener la capacidad de enseñar el valor agregado de las soluciones informáticas, que respondan a las preguntas: ¿por qué lo estamos haciendo? y ¿cuáles son las ventajas de uso de estos sistemas? La asesoría personalizada también debería ser implementada y evaluada. Además, establecer redes de colaboración es importante a nivel local (entre universidades y instituciones) pero también con instituciones internacionales (universidades y centros de investigación). Asimismo, es útil revisar las experiencias de otros programas y aprender de ellos. Es decir, evaluar que es lo que sí o no funcionó y aprender de estas experiencias para poder evitar o poder expandir estas lecciones y aplicarlas a nuestro medio.

Además, tiene que haber un proceso sistemático de integración, los que serán los mecanismos de nivelación para personas de diferentes disciplinas. Una propuesta es ofrecer un curso de introducción a la informática biomédica que incluya bases de datos y conceptos de programación para los profesionales de la salud y, a su vez, un curso de introducción a las ciencias biológicas, tales como terminologías en salud y temas de salud global para profesionales relacionados con las ciencias de la computación e ingeniería. También se discutió que los temas comunes para todos los profesionales debieran incluir: procesos asistenciales tales como la gestión y un curso de administración. Además, debería existir también un énfasis en la formación en investigación, que podría incluir temas como metodología de la investigación, búsqueda bibliográfica en bases de datos, lectura crítica de la literatura, escritura de proyectos de investigación, ensayos clínicos y conceptos de ética de la investigación en humanos.

La evaluación de los alumnos y egresados no solo debe ser a corto plazo, sino además al largo plazo, para así determinar el impacto que han tenido los alumnos en su trabajo y su vida académica. El éxito de un estudiante se debe medir no solo en donde está trabajando en determinado momento, sino también en el número de publicaciones y proyectos financiados. Otro punto por tomar en 
cuenta es determinar los roles de las personas que vamos a entrenar. La asociación IMIA (International Medical Informatics Association) publicó a principios del año 2010, un documento revisado con recomendaciones acerca de las competencias que deben poseer los estudiantes con relación al entrenamiento en informática biomédica ${ }^{(19)}$.

\section{RECURSOS HUMANOS Y PERFIL DEL ESTUDIANTE}

Los recursos humanos necesarios provienen de las ciencias de la salud, ciencias sociales, informática y las ciencias básicas. Por lo tanto, estos profesionales podrían incluir por ejemplo a: médicos, odontólogos, biólogos, microbiólogos, enfermeras, informáticos, registradores, administradores, bioestadísticos, bibliotecólogos, epidemiólogos, psicólogos, sociólogos y antropólogos. Otro aspecto que se discutió en el taller, fue la necesidad de evaluación continua de los egresados de los programas de educación en informática biomédica. El programa AMAUTA ha reportado previamente la evaluación de los cursos cortos no solo al finalizar cada curso sino también mediante el seguimiento a seis meses de finalizado el curso ${ }^{(11)}$.

El perfil de los candidatos a nivel de posgrado incluye: ser competentes en inglés y tener un conocimiento similar al de la Internacional Computer Driving License (ICDL). Además, el solicitante deberá reunir otras cualidades como tener una mente inquisitiva, iniciativa, imaginación y ser inquieto intelectualmente.

\section{PRIORIDADES DE INVESTIGACIÓN EN INFORMÁTICA BIOMÉDICA}

Se discutió la necesidad de desarrollar nuestras propias evidencias, ya que los resultados encontrados en otros países no podrían aplicarse necesariamente a nuestro contexto, pues tienen diferentes necesidades y cultura. Las áreas de investigación discutidas fueron amplias: salud pública, gestión en salud, telemedicina, salud móvil (mHealth), bioinformática, medicina, enfermería y toma de decisiones. Además, a fin de promover nuestras actividades y aprender de estas experiencias, hay que aprender a escribir propuestas de subvención y escribir artículos científicos. También tenemos que formar alianzas de investigación.

\section{MECANISMOS DE FINANCIACIÓN PARA LOS PROGRAMAS DE ENTRENAMIENTO E INVESTIGACIÓN EN INFORMÁTICA BIOMÉDICA}

Los mecanismos de financiación deben centrarse en la obtención de subvenciones, pero se discutió la necesidad de encontrar mecanismos para la sostenibilidad. Esto puede ser obtenido a través de fundaciones, fuentes del gobierno (a través del Ministerio de Salud, Ministerio de Educación, Ministerios de Transportes y Comunicaciones, etc.), sector público y privado. También se deben promover el desarrollo de patentes y, en el caso de trabajar con programas de código abierto, se puede desarrollar los mecanismos de "Software como Servicio" (Software as a Service, SaaS).

\section{EQUIPO MULTIDISCIPLINARIO}

Se concluyó que en cada país la conformación de equipos es diferente. Por ejemplo, en Uruguay el equipo multidisciplinario se compone de médicos, secretarios, enfermeras y administradores. En Colombia está compuesto de ingenieros, antropólogos, enfermeras, y biólogos. Ecuador cuenta con ingenieros y médicos que desarrollan proyectos de telemedicina en zonas rurales. Perú cuenta con una amplia gama de profesionales que incluyen a físicos, matemáticos, bioestadísticos, ingenieros de sistemas, biólogos, obstetrices y médicos.

\section{DISCUSIÓN}

Hemos descrito la experiencia del primer taller internacional de expertos en informática biomédica en la región andina que revisa nueve casos de estudio y discute la necesidad de entrenamiento e investigación multidisciplinaria en informática biomédica en áreas prioritarias para América Latina.

Uno de los aspectos que se discutió con profundidad, fueron los desafíos que tienen que enfrentar los profesionales de salud en América Latina para el uso y aplicación eficiente de las TIC. Por ejemplo, los bajos recursos, la sobresaturación de tiempo y el poco tiempo para tomar cursos de entrenamiento, son algunas de las barreras. Similares situaciones se ha reportado en otras regiones, como África del Este. Por ejemplo, en una encuesta realizada por Braa et al. en Mozambique, determinaron que el principal obstáculo para el uso de las TIC en salud fue un bajo nivel de calificación y de educación en el manejo de sistemas informáticos aplicados a la salud ${ }^{(20)}$

Los talleres en grupo reflejaron que los países de América Latina comparten los mismos retos y necesidades. Además, es importante definir competencias para los estudiantes y se tiene que determinar qué método de enseñanza se aplica mejor en nuestro medio. Brasil es un buen ejemplo; las universidades brasileñas han tenido éxito en 
atraer a profesionales de diferentes áreas ${ }^{(6,21,22)} \mathrm{y}$, en muchos casos, cada estudiante tiene un supervisor principal y un cosupervisor. Esto permite estimular al estudiante provocando una experiencia enriquecedora para el estudiante y el profesorado. El trabajo en equipo y los proyectos en equipo son esenciales y permiten la promoción de las colaboraciones sur-sur, muy necesarias en nuestra región.

Además, en muchas situaciones sucede que los recursos humanos están pobremente capacitados en el uso de tecnologías para el cuidado de la salud. Braa y Muquinge han señalado que debido a los escasos recursos humanos y los pobres conocimientos sobre las mejores prácticas de desarrollo de sistemas informáticos en salud, no se puede realmente aprovechar el potencial que las TIC pueden ofrecer en el campo de la salud ${ }^{(23)}$.

A pesar que sabemos que impartir cursos cortos es una estrategia popular en muchos países de América Latina, existen otras estrategias de aprendizaje como los sistemas de e-learning usados en otros países ${ }^{(24)}$.

Finalmente, hay que mencionar la importancia de las colaboraciones entre países desarrollados y países en desarrollo, y también dentro de los propios países en desarrollo, como se ha visto en una serie de proyectos que han sido mencionados en los casos de estudio (por ejemplo, el programa AMAUTA). Este tipo de colaboraciones son promovidas en América Latina a través de la Federación Regional de Informática de la Salud para América Latina y el Caribe (IMIA-LAC). Podemos promover no solo la colaboración norte-sur, sino también la colaboración sur-sur, como se ha hecho, por ejemplo, en el campo de las tecnologías móviles aplicadas a la salud, o mHealth ${ }^{(25)}$.

\section{LA RED QUIPU}

Como resultado de la reunión y la necesidad de formar colaboraciones nació la Red QUIPU. Esta Red se ha establecido debido a la necesidad de ampliar y consolidar una red de investigación para vincular a los investigadores en la región andina, de América Latina y universidades e instituciones de los Estados Unidos y el extranjero. Esta red incluye representantes del programa Fogarty Informatics Training for Global Health (7). La página web de la QUIPU Red estará disponible en el siguiente enlace: http://red.andeanquipu.org. El sitio web incluirá información sobre las actividades y las instituciones colaboradoras de la Red QUIPU.

Tenemos el propósito de fortalecer la Red QUIPU añadiendo más socios e instituciones de países de
América Latina y en el extranjero. La idea de la Red QUIPU es expandir y consolidar una red de investigación en la región andina, promoviendo la colaboración sursur, y de las universidades e instituciones colaboradoras a nivel global.

La Red QUIPU, con la ayuda de sus colaboradores, espera extender la visión multidisciplinaria de la salud global y la investigación en informática biomédica en América Latina. La teoría y práctica se vincularán a través del centro QUIPU con la ayuda de herramientas de entrenamiento que serán ofrecidas de manera presencial y a distancia para los investigadores, profesores y estudiantes a través de diferentes herramientas en línea, como boletines, página web, páginas wiki y blogs. El uso de estas nuevas tecnologías en entrenamiento y la investigación en informática biomédica será fundamental para mejorar la salud de las poblaciones en la región andina y en todo el mundo.

\section{AGRADECIMIENTOS}

Agradecemos a todos los participantes nacionales e internacionales del Taller Internacional, en especial a los miembros del "Scientific Advisory Board" del proyecto QUIPU que participaron en el taller: Oscar Feo (Organismo Andino de Salud); Sherrilynne Fuller (University of Washington); Robert Gilman (Johns Hopkins University); Arthur Gruber (Universidad de São Paulo); Humberto Guerra (Universidad Peruana Cayetano Heredia); Henry Harman (Fulbright Perú); King Holmes (University of Washington); Ann Marie Kimball (University of Washington); Peter Myler (University of Washington); y Jiajie Zhang (University of Texas - Houston). Agradecemos también al equipo organizador, en particular a la Lic. Melina Zevallos, coordinadora del proyecto QUIPU, y a la Lic. Claudia Morales de la UPCH.

\section{MIEMBROS DE LA RED QUIPU}

Osmán J. Argüello (Asociación Venezolana de Informática en Salud, Venezuela); Daniel Capurro (Universidad Católica de Chile, Chile); Alvaro Margolis (Federación Médica del Interior, Uruguay); María T. Mijares (Telemedicina, Ecuador); Paula Otero (Hospital Italiano de Buenos Aires, Argentina); Juan Puyana (Universidad Javeriana, Colombia y University of Pittsburgh Medical Center); Álvaro Rendón y Carlos Hernán Sierra (Universidad del Cauca, Colombia); Investigadores del Proyecto QUIPU: Magaly M. Blas (Universidad Peruana Cayetano Heredia, Perú); Cesar P. Cárcamo (Universidad Peruana Cayetano Heredia, Perú); Walter H. Curioso (Universidad Peruana Cayetano 
Heredia, Perú); Patricia J. García (Universidad Peruana Cayetano Heredia, Perú); Andrés G. Lescano (US Naval Medical Research Center Detachment, Peru); Diego M. López (Universidad del Cauca, Colombia); Mirko Zimic (Universidad Peruana Cayetano Heredia, Perú).

\section{Fuente de Financiamiento}

Este trabajo contó con el apoyo del Proyecto QUIPU - UPCH, un programa auspiciado por el Fogarty International Center/National Institutes of Health (FIC/ $\mathrm{NIH}$ ), proyecto: D43TW008438 y parcialmente por el proyecto R01TW007896 financiado por el FIC.

\section{Conflictos de Interés}

Los autores declaran no tener conflictos de interés en la publicación de este artículo.

\section{REFERENCIAS BIBLIOGRÁFICAS}

1. Blaya JA, Fraser HS, Holt B. E-health technologies show promise in developing countries. Health Aff (Millwood). 2010;29(2):244-51.

2. World Health Organization. eHealth Resolution, 58th World Health Assembly, Resolution 21. Geneva: WHO, 2005.

3. Curioso WH, Segovia Juárez J. Informática biomédica: oportunidades para la educación, investigación y desarrollo en el Perú. En: Chocobar M, Gotuzzo E, Gozzer E, Jiménez F, Murrugarra L, Zubko A (eds). Perú y América Latina en la era digital: transformando la sociedad. Lima: Universidad Peruana Cayetano Heredia; 2007

4. Gatewood L, Limburg M, Gardner R, Haux R, Jaspers $\mathbf{M}, \mathbf{S c h m i d t} \mathbf{D}$, et al. International master classes in health informatics. Int J Med Inform. 2004;73(2):111-16.

5. Seebregts CJ, Mamlin BW, Biondich PG, Fraser HS, Wolfe BA, Jazayeri D, et al. The OpenMRS Implementers Network. Int J Med Inform. 2009;78(11):711-20.

6. Marin HF, Massad E, Marques EP, Azevedo RS, OhnoMachado L. Training health informatics professionals in Brazil: rationale for the development of a new certificate program. AMIA Annu Symp Proc. 2005:1042.

7. Fogarty International Center: Informatics Training for Global Health (ITGH) [Página en Internet]. Bethesda, MA: FIC; 2010. [Fecha de acceso: 10 de julio de 2010] Disponible en: http://www.fic.nih.gov/programs/training_ grants/itgh/

8. Curioso WH, Castagnetto J, Lazo-Escalante M, Peinado J, Rubio CF. eHealth in Peru: A country case study. In: Making the eHealth connection: global partnerships, local solutions. Bellagio: Rockefeller Foundation; 2008.

9. Curioso WH, Fuller S, Garcia PJ, Holmes KK, Kimball AM. Ten years of international collaboration in biomedical informatics and beyond: the AMAUTA program in Peru. $\mathrm{J}$ Am Med Inform Assoc. 2010;17(4):477-480

10. Karras BT, Kimball AM, Gonzales V, Pautler NA, Alarcon J, Garcia PJ, et al. Informatics for Peru in the new millennium. Stud Health Technol Inform. 2001;84:1033-37.

11. Curioso WH, Hansen JR, Centurion-Lara A, Garcia PJ, Wolf FM, Fuller S, et al. Evaluation of a joint Bioinformatics and Medical Informatics international course in Peru. BMC Medical Education. 2008; 8:1

12. Blas MM, Curioso WH, Alva IE, Carcamo C, SegoviaJuarez JL, Garcia PJ, et al. Evaluación de un curso internacional y multidisciplinario de informática biomédica en el perú. Buenos Aires: INFOLAC; 2008.

13. Zimic M, Coronel J, Gilman RH, Luna CG, Curioso WH, Moore DA. Can the power of mobile phones be used to improve tuberculosis diagnosis in developing countries? Trans R Soc Trop Med Hyg. 2009;103(6):638-40.

14. Rey-Moreno C, Reigadas JS, Villalba EE, Vinagre JJ, Fernández AM. A systematic review of telemedicine projects in Colombia. J Telemed Telecare. 2010;16(3):114-19.

15. Gonzalez Bernaldo de Quiros F, Luna D, Otero P, Baum A, Borbolla D. Spreading knowledge in medical informatics: the contribution of the Hospital Italiano de Buenos Aires. Yearb Med Inform. 2009;:147-52.

16. Otero P, Hersh W, Luna D, González Bernaldo de Quirós F. A medical informatics distance-learning course for Latin America. Translation, implementation and evaluation. Methods Inf Med. 2010;49(3):310-15.

17. Margolis A, Vero1 A, Bessonart L, Barbiel A, Ferla M. Health information systems training for a countrywide implementation in uruguay. Methods Inf Med. 2009(1):153-57.

18. Capurro D. Health informatics in Chile: responding to health reforms. Health Info Libr J. 2007;24(4):287-91.

19. Mantas J, Ammenwerth E, Demiris G, Hasman A, Haux R, Hersh W, et al. Recommendations of the International Medical Informatics Association (IMIA) on education in biomedical and health informatics. First revision. Methods Inf Med. 2010;49(2):105-20.

20. Braa J, Macome E, DaCosta JL, Mavimbe JC, Nhampossa JL, José B, et al. A study of actual and potential usage of information and communication technologies at district and provincial levels in Mozambique with a focus on the health sector. Electron J Inform Syst Dev Ctries. 2001;5(2):1-29.

21. Marques EP, Marin HF, Massad E, Fraser H, OhnoMachado L. Training in health informatics in Brazil. Stud Health Technol Inform. 2002;90:757-760

22. Marin HF, Massad E, Marques EP, Ohno-Machado L. International training in health informatics: a Brazilian experience. Stud Health Technol Inform. 2004;107(Pt 2): 898-902.

23. Braa J, Muquinge H. Building collaborative networks in Africa on health information systems and open source software development - experiences from the HISP/ BEANISH Network. In: Health Informatics in Africa HELINA: E-Health in Africa. Bamako, Mali: HELINA; 2007.

24. Montenegro S, Taliercio V, Otero P, Luna D, Wassermann $S$, Magallan L, et al. Analysis of dropouts in the e-learning courses at the Virtual Campus of the Hospital Italiano de Buenos Aires. Buenos Aires: INFOLAC. 2008.

25. Curioso WH, Mechael PN. Enhancing 'M-health' with south-tosouth collaborations. Health Aff (Millwood). 2010;29(2): 264-67.

Correspondencia:Dr. Walter H. Curioso

Dirección: Av. Honorio Delgado 430,Lima 31, Perú

Teléfono: (511) 319-0028

Correo electrónico: walter.curioso@upch.pe 